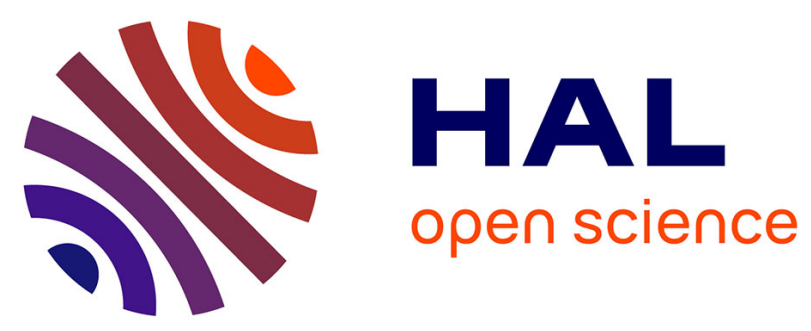

\title{
Dynamic Magnetostriction of CoFe2O4 and Its Role in Magnetoelectric Composites
}

\author{
Alex Aubert, V. Loyau, Yoann Pascal, F. Mazaleyrat, M. Lobue
}

\section{To cite this version:}

Alex Aubert, V. Loyau, Yoann Pascal, F. Mazaleyrat, M. Lobue. Dynamic Magnetostriction of $\mathrm{CoFe} 2 \mathrm{O} 4$ and Its Role in Magnetoelectric Composites. Physical Review Applied, 2018, 9 (4), pp.044035. 10.1103/PhysRevApplied.9.044035 . hal-01782289

\section{HAL Id: hal-01782289 \\ https://hal.science/hal-01782289}

Submitted on 1 May 2018

HAL is a multi-disciplinary open access archive for the deposit and dissemination of scientific research documents, whether they are published or not. The documents may come from teaching and research institutions in France or abroad, or from public or private research centers.
L'archive ouverte pluridisciplinaire HAL, est destinée au dépôt et à la diffusion de documents scientifiques de niveau recherche, publiés ou non, émanant des établissements d'enseignement et de recherche français ou étrangers, des laboratoires publics ou privés. 


\title{
Dynamic Magnetostriction of $\mathrm{CoFe}_{2} \mathrm{O}_{4}$ and Its Role in Magnetoelectric Composites
}

\author{
A. Aubert, ${ }^{*}$ V. Loyau, Y. Pascal, F. Mazaleyrat, and M. LoBue \\ SATIE UMR 8029 CNRS, ENS Paris-Saclay, Université Paris-Saclay, \\ 61 avenue du Président Wilson, 94235 Cachan Cedex, France
}

(Received 20 November 2017; published 24 April 2018)

\begin{abstract}
Applications of magnetostrictive materials commonly involve the use of the dynamic deformation, i.e., the piezomagnetic effect. Usually, this effect is described by the strain derivative $\partial \lambda / \partial H$, which is deduced from the quasistatic magnetostrictive curve. However, the strain derivative might not be accurate to describe dynamic deformation in semihard materials as cobalt ferrite (CFO). To highlight this issue, dynamic magnetostriction measurements of cobalt ferrite are performed and compared with the strain derivative. The experiment shows that measured piezomagnetic coefficients are much lower than the strain derivative. To point out the direct application of this effect, low-frequency magnetoelectric (ME) measurements are also conducted on bilayers $\mathrm{CFO} / \mathrm{Pb}(\mathrm{Zr}, \mathrm{Ti}) \mathrm{O}_{3}$. The experimental data are compared with calculated magnetoelectric coefficients which include a measured dynamic coefficient and result in very low relative error $(<5 \%)$, highlighting the relevance of using a piezomagnetic coefficient derived from dynamic magnetostriction instead of a strain derivative coefficient to model ME composites. The magnetoelectric effect is then measured for several amplitudes of the alternating field $H_{\mathrm{ac}}$, and a nonlinear response is revealed. Based on these results, a trilayer $\mathrm{CFO} / \mathrm{Pb}(\mathrm{Zr}, \mathrm{Ti}) \mathrm{O}_{3} / \mathrm{CFO}$ is made exhibiting a high magnetoelectric coefficient of $578 \mathrm{mV} / \mathrm{A}$ (approximately $460 \mathrm{mV} / \mathrm{cm} \mathrm{Oe}$ ) in an ac field of $38.2 \mathrm{kA} / \mathrm{m}$ (about $48 \mathrm{mT}$ ) at low frequency, which is 3 times higher than the measured value at $0.8 \mathrm{kA} / \mathrm{m}$ (approximately $1 \mathrm{mT}$ ). We discuss the viability of using semihard materials like cobalt ferrite for dynamic magnetostrictive applications such as the magnetoelectric effect.
\end{abstract}

DOI: 10.1103/PhysRevApplied.9.044035

\section{INTRODUCTION}

Magnetostriction is defined as the change in length of materials under the influence of an external magnetic field. The magnetostriction coefficient $\lambda$ is generally measured using the usual quasistatic strain-gauge technique, which gives the differential strain as a function of the bias magnetic field $H_{\mathrm{dc}}$. It has been shown that magnetostriction is a quadratic function of $M$, i.e., $\lambda \propto M^{2}$ [1-3]. Today, magnetostrictive materials are used in actuators [4,5], sonar transducers [6], motors [7], and various types of sensors $[8,9]$. The interest in oxide-based magnetostrictive materials such as cobalt ferrites (CFOs) has also increased due to their low cost, ease of fabrication, high resistivity, and high mechanical robustness compared to rare-earth intermetallics like Terfenol- $D$ [10].

Another advantage of cobalt ferrites is that their magnetostrictive properties can be tuned by inducing a uniaxial anisotropy. This can be done by magnetic annealing [11-15], magnetic-field-assisted compaction [16,17], or reaction under uniaxial pressure [18]. Another way to tune magnetostrictive properties of $\mathrm{CoFe}_{2} \mathrm{O}_{4}$ is by substitution of the $\mathrm{Fe}$ atoms by $\mathrm{Mg}, \mathrm{Al}, \mathrm{Ti}, \mathrm{Mn}, \mathrm{Ni}, \mathrm{Cu}, \mathrm{Zn}, \mathrm{Ga}, \mathrm{Zr}, \mathrm{Nb}$,

"alex.aubert@satie.ens-cachan.fr
In, etc., or even rare-earth elements [19-33]. Moreover, there is an increasing interest in synthesizing cobalt ferrite from recycled $\mathrm{Li}$-ion batteries to use it in magnetostrictive applications [25,34-36]. Hence, research in cobalt ferrites is very active, with a special focus on tuning their magnetostrictive properties.

Recently, these materials have also attracted renewed interest because of their potential use in extrinsic multiferroics, and particularly in magnetoelectric (ME) composites for smart electronic applications [37-41]. Indeed, cobalt ferrites are very attractive for this purpose and have been used either in laminated $[15,42-49]$ or in particulate [50-55] composites. The direct ME effect consists of a change in the electric polarization induced by a magnetic field. In ME heterostructures, the magnetoelectric coupling is due to the magnetic-mechanical-electric conversion through the interface between the two phases. This coupling results from the variation of the polarization in the piezoelectric layer caused by the dynamic mechanical deformation of the ferromagnet, i.e., the dynamic magnetostriction, induced by an alternating field $H_{\mathrm{ac}}$ superimposed to a bias magnetic field $H_{\mathrm{dc}}$. Hence, the ME effect arises mainly from the dynamic magnetostriction, i.e., the piezomagnetic coefficient of the magnetic material.

As stated by du Trémolet de Lacheisserie [1], the piezomagnetic coefficient is defined as the strain oscillation 
induced by an alternating magnetic field $H_{\mathrm{ac}}$ at any polarizing field $H_{\mathrm{dc}}$, which can be written $q^{\text {ac }}=$ $\left.\left(\partial \lambda_{\mathrm{ac}} / \partial H_{\mathrm{ac}}\right)\right|_{H_{\mathrm{dc}}}$, with $\lambda_{\mathrm{ac}}$ being the dynamic magnetostriction. It has also been modeled analytically by some authors [56-58] based on Gibbs free energy. However, the piezomagnetic coefficient is commonly extrapolated from the strain derivative of the quasistatic magnetostrictive curve, namely, $q^{\mathrm{dc}}=\partial \lambda / \partial H_{\mathrm{dc}}$ [59], and is usually considered the main parameter characterizing the magnetostrictive materials. Nevertheless, the static strain derivative $q^{\mathrm{dc}}$ may hardly be used to extrapolate the piezomagnetic coefficient $q^{\text {ac }}$ in all materials. Actually, the strain derivative $q^{\mathrm{dc}}$ is, by definition, related to the differential susceptibility of the material $\chi_{\text {diff }}$ [60], whereas the dynamic magnetostriction-and hence the piezomagnetic coefficient $q^{\text {ac }}$-should mainly depend on the dynamic susceptibility $\chi_{\mathrm{ac}}$ [61] of the material for any given bias magnetic field. This also means that dynamic magnetostriction should be correlated to the amplitude of the alternating field $H_{\text {ac }}$ because $\chi_{\text {ac }}$ depends on the amplitude of the minor loop. Hence, the strain derivative does not take into account the dynamic aspect but rather only the quasistatic differential strain, pointing out a possible inaccurate way of characterizing magnetostrictive materials for a dynamic applications purpose.

On the other hand, the piezoelectric coefficient $d_{33}$ for piezoelectric materials is always characterized by dynamic measurements, with the most common methods being the impedance resonance measurement or the laser interferometry [62]. Several decades ago, dynamic measurement was also used to investigate magnetostrictive Terfenol- $D$ alloys to show their potential as transducers. These measurements were performed either with a strain gauge [63-65], an accelerometer [66], a laser-Dopplervibrometry system $[67,68]$ or by using a three-terminal capacitance technique [69].

Nevertheless, to the best of our knowledge, the dynamic magnetostriction of cobalt ferrite has not been investigated. Yet the approximation of considering the static strain derivative $q^{\mathrm{dc}}$ as a relevant parameter to describe the dynamic magnetostriction might be particularly misleading for semihard materials such as cobalt ferrite. In fact, as suggested by Srinivasan [70], this material exhibits large anisotropy, which limits domain rotation and hence dynamic deformation, while this material exhibits very high saturation magnetostriction $\left(\lambda_{S}>300 \mathrm{ppm}\right)$ [71]. This anisotropy could explain why the magnetoelectric effect is much higher for materials with high permeability and low magnetostriction saturation $\left(\lambda_{S}\right)$ such as nickel ferrite (NFO), nickel zinc ferrite (NZFO), and manganese zinc ferrite (MZFO) than for materials with low permeability and high $\lambda_{S}$ such as cobalt ferrite [72-76]. Yet the strain derivative $q^{\mathrm{dc}}$ is higher for CFO than for NFO and MZFO, showing that this parameter may not fully and accurately characterize the ME effect. This approximation also had consequences in the modeling of the magnetoelectric effect, where authors had to introduce a very low mechanical coupling factor for ME heterostructures including CFO to fit predicted values with experimental data, which was not the case for NFO and NZFO [72,77].

In this paper, we measure the dynamic magnetostriction of the cobalt ferrite using a dynamic strain-gauge experiment described in Sec. II B. We perform measurements on an isotropic and anisotropic cobalt ferrite disk $(2 \mathrm{~mm}$ in thickness, $10 \mathrm{~mm}$ in diameter) and obtain a longitudinal piezomagnetic coefficient $q_{11}^{\text {ac }}$, at any working point $H_{\mathrm{dc}}$. We investigate the influence of the amplitude of the alternating field $H_{\mathrm{ac}}$ from $0.8 \mathrm{kA} / \mathrm{m}(1 \mathrm{mT})$ to $76.4 \mathrm{kA} / \mathrm{m}$ (96 mT) and find a high dependence of $q_{11}^{\text {ac }}$ on it. We compare these values with the usual quasistatic strain derivative coefficient $q_{11}^{\mathrm{dc}}$ and find them to be much lower: $80 \%$ lower at $0.8 \mathrm{kA} / \mathrm{m}$ and $40 \%$ lower at the optimum excitation field of $50.9 \mathrm{kA} / \mathrm{m}$ for the anisotropic sample. Based on these results, the piezomagnetic coefficients derived from dynamic magnetostriction measurements are introduced in a low-frequency transverse ME model, and the calculated values are compared with experimental data retrieved for a ME bilayer CFO/PZT. A good accuracy is found between the predicted and experimental data (relative error $<5 \%$ ). Moreover, a strong dependence of the ME coefficient on the amplitude of $H_{\mathrm{ac}}$ is described, pointing out the importance of choosing the accurate driving field to optimize the ME effect for heterostructures composed of cobalt ferrite. Finally, by making a ME trilayer with optimized geometry, we are able to reach a maximum ME coefficient of $578 \mathrm{mV} / \mathrm{A}$ (approximately $460 \mathrm{mV} / \mathrm{cm} \mathrm{Oe}$ ) at low frequency with an ac field of $38.2 \mathrm{kA} / \mathrm{m}$. Considering the results, we will discuss the viability of using cobalt ferrite for a magnetoelectric purpose.

\section{EXPERIMENT}

\section{A. Sample preparation}

Two different kinds of polycrystalline cobalt ferrite are prepared, one exhibiting isotropic properties and the other presenting a uniaxial anisotropy. In both cases, nanosized $(<50 \mathrm{~nm})$ oxides $\mathrm{Fe}_{2} \mathrm{O}_{3}$ and $\mathrm{Co}_{3} \mathrm{O}_{4}$ (Sigma-Aldrich) are used as precursors in a molar ratio of $3: 1$. Powders are mixed in a planetary ball mill for $30 \mathrm{~min}$ at $400 \mathrm{rpm}$, then ground for $1 \mathrm{~h}$ at $600 \mathrm{rpm}$. In the first method, the synthesis of the spinel phase is achieved by the usual calcination at $900^{\circ} \mathrm{C}$ for $12 \mathrm{~h}$. After grinding the cobalt ferrite powder at $550 \mathrm{rpm}$ for $1 \mathrm{~h}$, it is sintered using spark plasma sintering (SPS) in the same conditions as described elsewhere [18]. Using this process permits us to obtain isotropic cobalt ferrite. In the second method, the oxide mixture is processed by SPS, where both the synthesis and the sintering are performed (reactive sintering). This technique is found to introduce a uniaxial anisotropy to cobalt ferrite in the direction of the applied pressure (for details, see Ref. [18]). 
Both methods result in cobalt ferrite with a large majority spinel phase $(>91 \%)$, and the final samples are identical cylindrical pellets $10 \mathrm{~mm}$ in diameter and $2 \mathrm{~mm}$ thick with a high relative density (about 97\%). We refer to the isotropic cobalt ferrite as CFOI, and the anisotropic one as CFOA.

To manufacture magnetoelectric composites, cobalt ferrite disks are bonded on 1-mm-thick and 10-mmdiameter commercial lead zirconate titanate (PZT) disks (Ferroperm PZ27) using silver epoxy (EPO-TEK E4110). The piezoelectric samples are polarized along the thickness direction. The magnetoelectric bilayers are, finally, disks with a thickness of $3 \mathrm{~mm}$ and a diameter of $10 \mathrm{~mm}$. Measurements are also performed on a trilayer CFO/PZT/ CFO with layer thicknesses of $0.4 \mathrm{~mm} / 0.75 \mathrm{~mm} / 0.4 \mathrm{~mm}$, and with a diameter of $10 \mathrm{~mm}$.

\section{B. Experimental setup and methods}

Magnetic measurements are carried out with a vibrating-sample magnetometer (Lakeshore 7400) up to a maximum field of $800 \mathrm{kA} / \mathrm{m}$. They are performed on ME bilayers along the radial direction to obtain the effective magnetization, which includes the radial demagnetizing field and the strain contribution from the bonded piezoelectric layer, as in magnetostrictive and magnetoelectric measurements.

Static magnetostriction measurements are performed at room temperature using the usual strain-gauge method [78], where deformation and resistance of the gauge are correlated by

$$
\frac{R_{H}-R_{0}}{R_{0}}=K \frac{l_{H}-l_{0}}{l_{0}}=K \lambda,
$$

where $R_{H}$ is the resistance of the gauge at any bias field $H_{\mathrm{dc}}, R_{0}$ the initial resistance for $H_{\mathrm{dc}}=0, K$ the gauge factor, $l_{H}$ the deformation at any bias field $H_{\mathrm{dc}}$, and $l_{0}$ the initial deformation for $H_{\mathrm{dc}}=0$.

Self-temperature-compensation semiconductor gauges (KYOWA KSN-2-120-E4-11) are used to obtain a high gauge factor $(K \sim 104)$ and a low induced magnetic-field voltage. The gauges are bonded on the pellet surface and the applied magnetic field is in the plane of the disk, either parallel $\left(\lambda_{11}\right)$ or perpendicular $\left(\lambda_{21}\right)$ to the gauge.

A dynamic magnetostriction measurement is also performed at room temperature using the same strain gauge. To measure the dynamic deformation, a low-frequency (80-Hz) alternating magnetic field $H_{\text {ac }}$ has to be superimposed on the static one, $H_{\mathrm{dc}}$. As defined previously, the piezomagnetic coefficient should be written

$$
q^{\mathrm{ac}}=\left(\frac{\partial \lambda_{\mathrm{ac}}}{\partial H_{\mathrm{ac}}}\right)_{H_{\mathrm{dc}}},
$$

where $\lambda_{\mathrm{ac}}$ is the dynamic magnetostriction, defined by

$$
\lambda_{\mathrm{ac}}=\frac{\Delta l_{\mathrm{ac}}}{l_{H}},
$$

where $\Delta l_{\mathrm{ac}}$ is the dynamic deformation and $l_{H}$ the deformation for any given bias field. The dynamic magnetostriction can be linked to the strain-gauge resistance by

$$
\lambda_{\mathrm{ac}}=\frac{\Delta R_{\mathrm{ac}}}{K R_{H}},
$$

where $\Delta R_{\mathrm{ac}}$ is the alternating resistance and $R_{H}$ the resistance for any given bias field. By combining Eqs. (2) and (4), we relate the piezomagnetic coefficient and the strain gauge:

$$
q^{\mathrm{ac}}=\left(\frac{1}{K R_{H}} \frac{\partial R_{\mathrm{ac}}}{\partial H_{\mathrm{ac}}}\right)_{H_{\mathrm{dc}}} .
$$

Hence, by imposing a dc current $I_{\mathrm{dc}}$ through the gauge, the previous equation can be expressed as

$$
q^{\mathrm{ac}}=\left(\frac{1}{I_{\mathrm{dc}} K R_{H}} \frac{V_{q}^{\mathrm{ac}}}{H_{\mathrm{ac}}}\right)_{H_{\mathrm{dc}}},
$$

where $V_{q}^{\mathrm{ac}}$ is the dynamic voltage at the terminals of the gauge, $I_{\mathrm{dc}}$ the current applied in the gauge, $K$ the gauge factor, $R_{H}$ the resistance of the gauge at any bias field $H_{\mathrm{dc}}$, and $H_{\mathrm{ac}}$ the alternating magnetic field.

Here, the aim is to measure the dynamic voltage, which is directly related to the dynamic deformation, by means of a lock-in amplifier (EG\&G Princeton 5210) with high input impedance (100 M $\Omega$ ). As dynamic deformation tends to be lower than quasistatic deformation, the voltage signal is expected to be weak, especially for a low excitation field $H_{\mathrm{ac}}$. Hence, to improve the sensitivity of the measured voltage $V_{q}^{\text {ac }}$ and according to Eq. (6), we need a gauge showing a high gauge factor $K$ and a high electrical resistance $R_{H}$ and allowing a high current $I_{\mathrm{dc}}$. Semiconductor gauges are used because they provide a high gauge factor of 104, which is 50 times higher than the usual resistive gauge, and allow a maximum dc current of $20 \mathrm{~mA}$. However, their resistance is quite low $(R \sim 125 \Omega)$ and these gauges exhibit high temperature dependence. To overcome this issue, self-temperaturecompensation gauges are used. The electrical circuit of the dynamic magnetostriction measurement is depicted in Fig. 1(a).

The dc current flowing through the gauge is supplied by an LT3092 (Linear Technology). The stability of the current with regard to the variation of the resistance is tested beforehand. The current is fixed to $15 \mathrm{~mA}$ to avoid the deterioration of the gauge. To bypass any offset in the voltage measurement, a high-pass filter (HPF) is connected in series by adding a capacitor $\left(C_{f} \sim 1.5 \mathrm{nF}\right)$. Induced voltage in the gauge should also be limited thanks to the use 
(a)

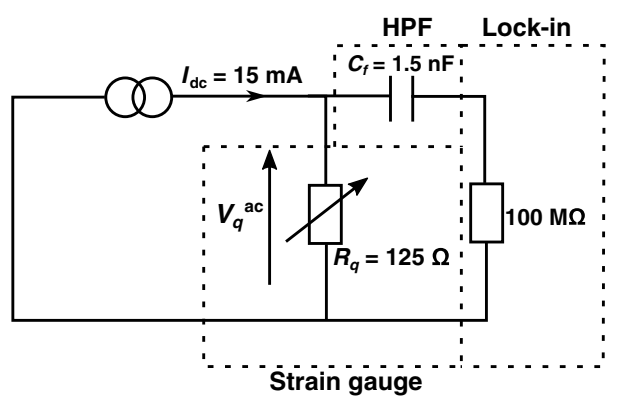

(b)

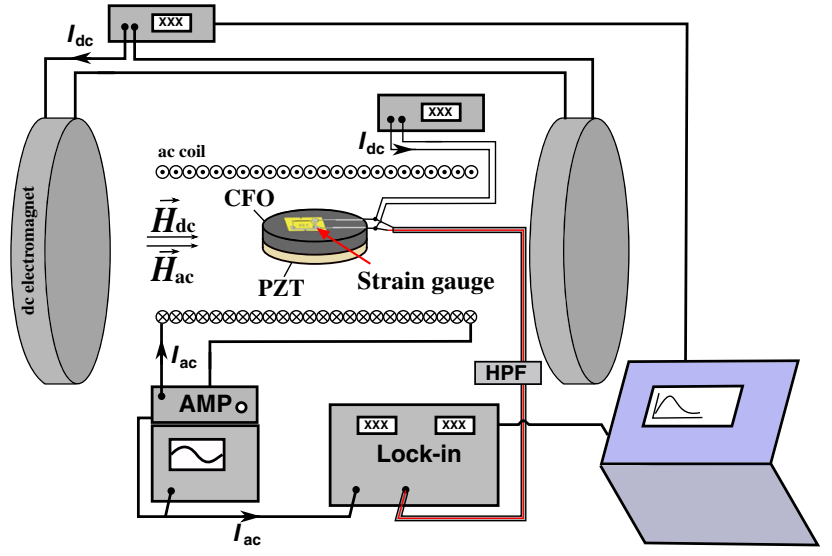

FIG. 1. (a) Electrical circuit of the dynamic magnetostriction measurement. (b) General setup of the dynamic deformation experiment.

of semiconductor gauges, which have a lower active surface than resistive gauges. The alternating field is produced by a solenoid where ac current is generated by a function generator connected to an amplifier (AMP), allowing us to reach amplitudes ranging from 0.8 to $76.4 \mathrm{kA} / \mathrm{m}$ ac rms. As a first approximation, in the calculation of Eq. (6), the $R_{H}$ value measured at $H_{\mathrm{dc}}=0 \mathrm{kA} / \mathrm{m}$ is kept constant instead of being retrieved at any given bias field. This approximation is rather good, as low error should be induced on the final value $(<0.5 \%)$. The general experimental setup is depicted in Fig. 1(b).

Magnetoelectric measurements are also performed by applying a low-frequency alternating field $H_{\mathrm{ac}}(80 \mathrm{~Hz})$ superimposed to a static field $H_{\mathrm{dc}}$. The alternating and static magnetic fields are produced by a solenoid and an electromagnet, respectively. Both fields are applied in plane [direction (1)], producing an electric field $E_{3}$ along the thickness direction (3) of the piezoelectric layer. The transversal magnetoelectric coefficient $\alpha_{31}$ can then be defined by

$$
\alpha_{31}=\frac{E_{3}}{H_{\mathrm{ac}}}=\frac{V_{3}}{t_{e} H_{\mathrm{ac}}},
$$

where $V_{3}$ is the magnetoelectric voltage at the terminals of the piezoelectric and $t_{e}$ the thickness of the piezoelectric. The magnetoelectric voltage is measured using a lock-in amplifier (EG\&G Princeton 5210) with high input impedance $(100 \mathrm{M} \Omega)$.

\section{RESULTS AND DISCUSSION}

\section{A. Magnetic properties and static magnetostriction}

The magnetic hysteresis loops of the two samples CFOI and CFOA are shown in Fig. 2 (the dotted lines). The anisotropic sample exhibits a higher coercive field $\left(H_{c}=51 \mathrm{kA} / \mathrm{m}\right)$ than the isotropic one $\left(H_{c}=18 \mathrm{kA} / \mathrm{m}\right)$. This is a consequence of the reaction under pressure which induces an easy axis along the thickness direction [18], and hence a higher anisotropy. The difference in the saturation magnetization is attributed to the presence of a secondary phase found in the samples [18]. After performing a major hysteresis loop, a recoil curve is measured that moves from remanence $(H=0 \mathrm{kA} / \mathrm{m})$ to saturation $(H=800 \mathrm{kA} / \mathrm{m})$ (the thick lines) in order to reproduce the same experimental conditions used in magnetostrictive and magnetoelectric measurements. The derivatives of both recoil curves are plotted in the inset of Fig. 2. The isotropic sample appears to be more sensitive to the field and exhibits a higher susceptibility than the anisotropic one, as the measurements are performed in the hard direction of CFOA. The maximum susceptibility appears for an applied field of 35 and $120 \mathrm{kA} / \mathrm{m}$ for CFOI and CFOA, respectively. This behavior should be attributed to the width of the hysteresis loops and plays a major role in the static and dynamic magnetostrictive curves.

Magnetostriction measurement of the isotropic and anisotropic cobalt ferrite are represented Figs. 3(a) and 3(b), respectively. For each sample, the strain derivatives along the longitudinal and the transverse direction are plotted, as is their sum. The isotropic sample shows the usual longitudinal and transverse magnetostriction behavior, with an approximate ratio of $2: 1$ between them at saturation. This isotropy

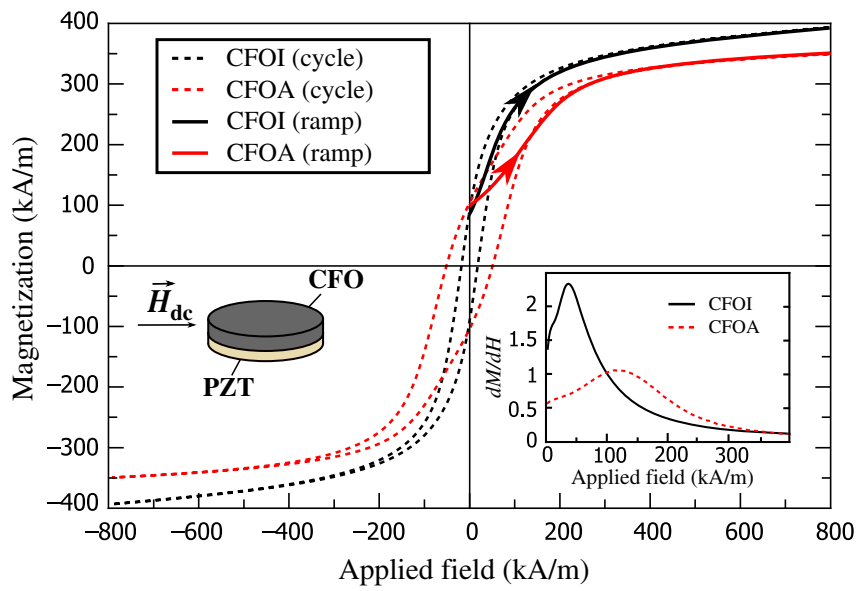

FIG. 2. Magnetic measurement of bilayers CFOI/PZT and $\mathrm{CFOA} / \mathrm{PZT}$. Measurements are performed in the radial direction (see the sketch), and dotted lines represent the $M-H$ hysteresis cycle. Thick lines symbolize the recoil curve progressing from 0 to $800 \mathrm{kA} / \mathrm{m}$ and are measured after performing the $M-H$ loops. (Inset) The derivative of the recoil curve with respect to the applied field. 

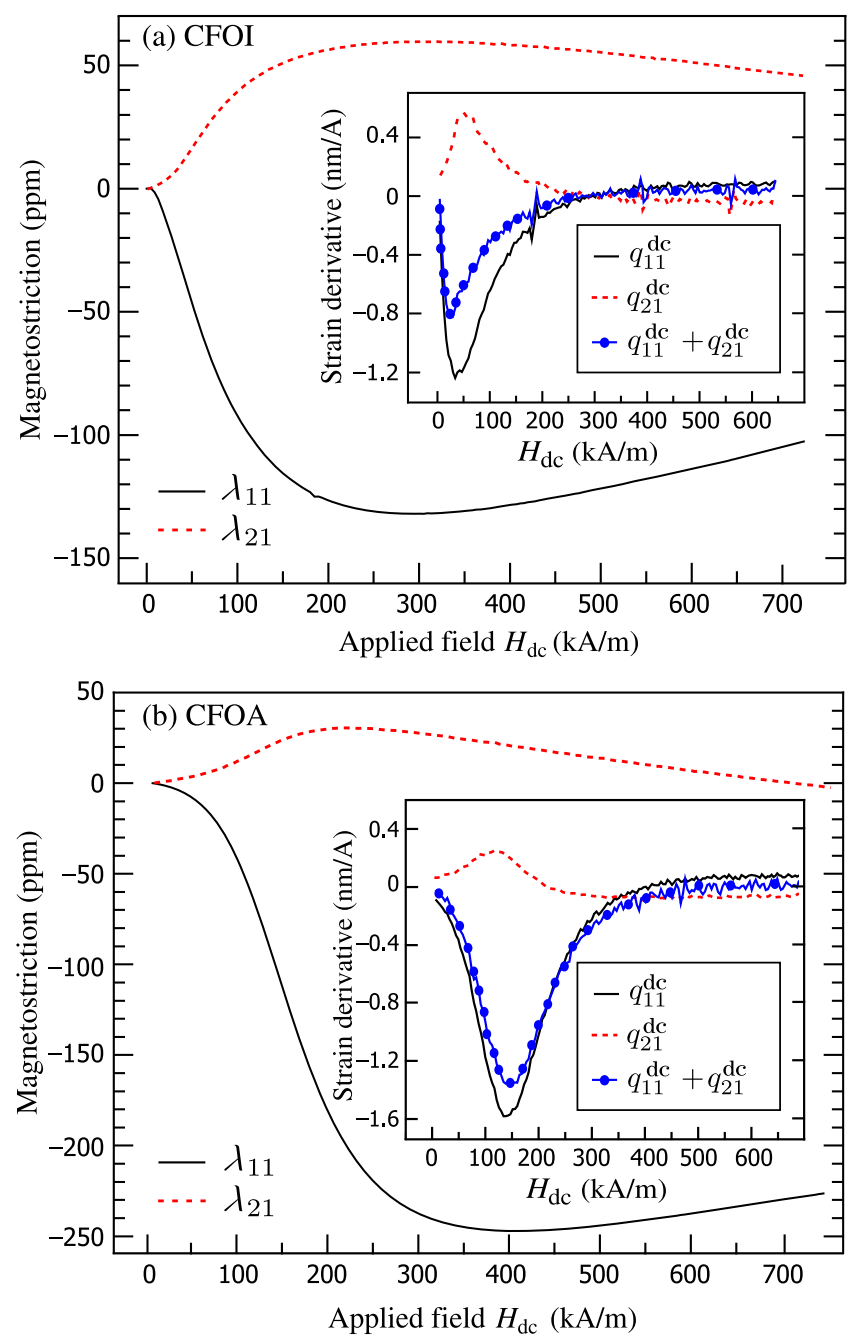

FIG. 3. Static longitudinal $\lambda_{11}$ and transversal $\lambda_{21}$ magnetostrictive curves for (a) CFOI and (b) CFOA. (Inset) The dc strain derivatives $q^{\mathrm{dc}}$ along the longitudinal and transversal directions, and the sum of the two.

results in a sum $q_{11}^{\mathrm{dc}}+q_{21}^{\mathrm{dc}} \sim-0.8 \mathrm{~nm} / \mathrm{A}$ being reduced by a factor of 1.5 compared to the maximum value of $q_{11}^{\mathrm{dc}} \sim-1.2 \mathrm{~nm} / \mathrm{A}$. On the other hand, the uniaxial anisotropy has strong consequences on the magnetostrictive behavior. The longitudinal magnetostriction $\lambda_{11}$ is increased, while the transverse magnetostriction $\lambda_{21}$ is decreased. This effect is also apparent in the strain derivative, where the longitudinal one, $q_{11}^{\mathrm{dc}} \sim-1.55 \mathrm{~nm} / \mathrm{A}$, is enhanced, while the transverse one, $q_{21}^{\mathrm{dc}} \sim 0.25 \mathrm{~nm} / \mathrm{A}$, is reduced. The low $q_{21}^{\mathrm{dc}}$ results in a sum, $q_{11}^{\mathrm{dc}}+q_{21}^{\mathrm{dc}} \sim-1.35 \mathrm{~nm} / \mathrm{A}$, much higher than that for the isotropic materials. This increase is of great relevance for improving the transverse magnetoelectric effect, which is known to depend on the sum $q_{11}+q_{21}$ $[42,72]$. However, the drawback of having anisotropic sample is the decrease of its susceptibility. In fact, as shown previously, CFOA requires a higher bias field $H_{\mathrm{dc}}$ to reach the optimum strain derivative. The maximum longitudinal strain derivative $q_{11}^{\mathrm{dc}}$ is reached for an applied polarizing field of 38 and $135 \mathrm{kA} / \mathrm{m}$ for CFOI and CFOA, respectively. These values are in good agreement with the ones found for the maximum differential susceptibility measured in the previous paragraph, and they can be explained by the definition of the strain derivative:

$$
\begin{aligned}
q^{\mathrm{dc}} & =\frac{\partial \lambda}{\partial H_{\mathrm{dc}}} \\
& =\frac{\partial \lambda}{\partial M} \frac{\partial M}{\partial H_{\mathrm{dc}}}, \\
q^{\mathrm{dc}} & =\frac{\partial \lambda}{\partial M} \chi_{\mathrm{diff}},
\end{aligned}
$$

where $\chi_{\text {diff }}$ here refers to the slope of the recoil curve, whereas it is usually defined on the initial magnetization curve [60].

\section{B. Dynamic magnetostriction}

Dynamic magnetostriction measurements are performed using the experimental setup presented in Sec. II B. First, a low-frequency $(80-\mathrm{Hz})$ and low-amplitude $(0.8-\mathrm{kA} / \mathrm{m})$ alternating field is superimposed on the static magnetic field. Measurements for the longitudinal piezomagnetic coefficient $q_{11}^{\text {ac }}$ are represented in Fig. 4 for CFOI and CFOA. To compare, quasistatic strain derivative coefficients $q_{11}^{\mathrm{dc}}$ are also plotted in the figure. For both samples, the dynamic coefficient $\left(q_{11}^{\text {ac }}\right)$ is much lower than that for the static coefficients $\left(q_{11}^{\mathrm{dc}}\right)$. In fact, the measured piezomagnetic coefficient is decreased to 0.36 and $0.30 \mathrm{~nm} / \mathrm{A}$ compared to the strain derivative for $\mathrm{CFOI}$ and CFOA, respectively. It is worth noting that the reduction is less drastic for the isotropic sample than for the anisotropic one. This might be a consequence of the large anisotropy and coercive field found for CFOA, which limits domain rotation, and hence dynamic deformation. This result also proves that high-permeability materials are more efficient for a dynamic purpose at low driving field.

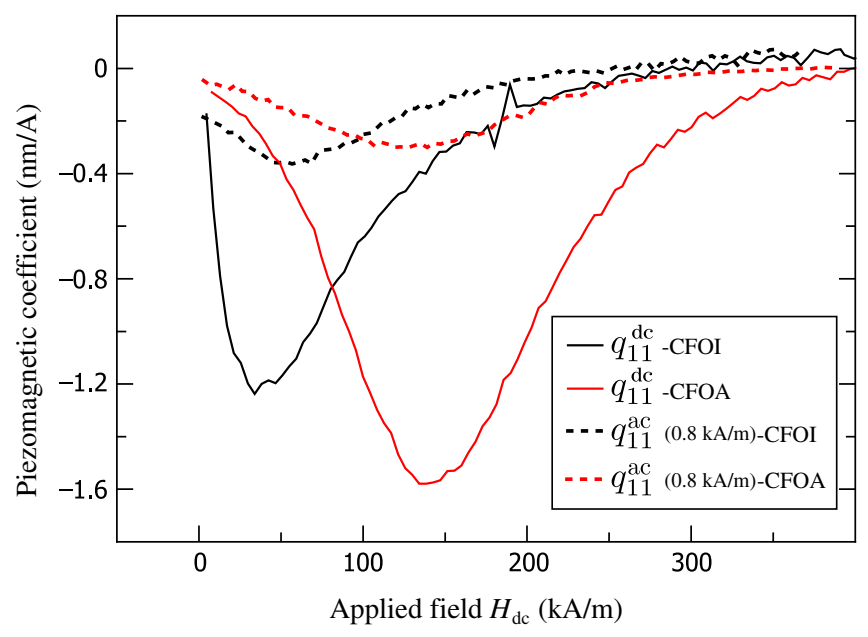

FIG. 4. Longitudinal strain derivative $q_{11}^{\mathrm{dc}}$ and piezomagnetic coefficient $q_{11}^{\text {ac }}\left(H_{\mathrm{ac}} \sim 0.8 \mathrm{kA} / \mathrm{m}\right)$ for CFOI and CFOA. 


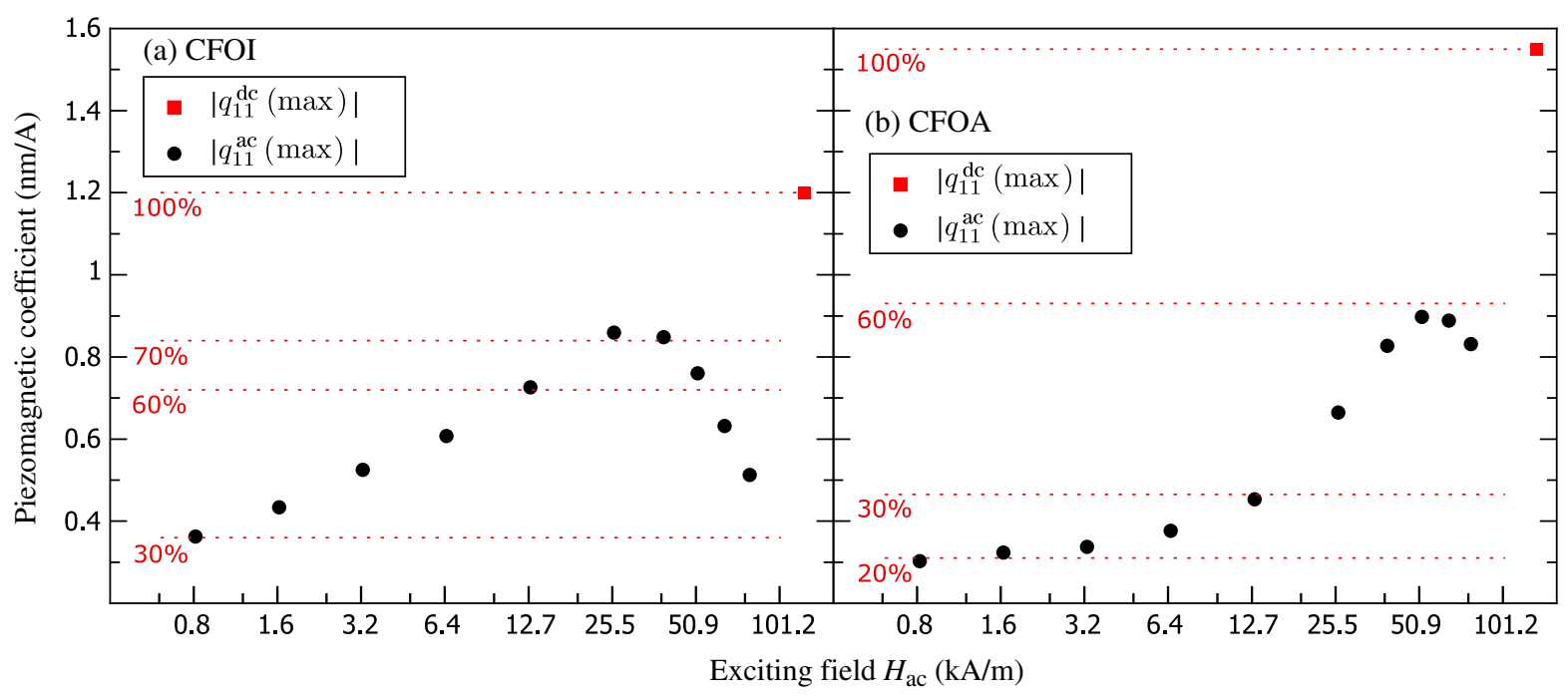

FIG. 5. Maximum longitudinal dynamic piezomagnetic coefficients $q_{11}^{\text {ac }}$ (modulus) as a function of the excitation field $H_{\text {ac }}$ for (a) CFOI and (b) CFOA (semilog 2 plot). Values are compared to the longitudinal strain derivative coefficient $q_{11}^{\mathrm{dc}}$.

To show the dependence of the piezomagnetic coefficient $q^{\text {ac }}$ on the amplitude of the alternating field, the same measurements are performed for several $H_{\mathrm{ac}}$ amplitudes: $0.8,1.6,3.2,6.4,12.7,25.5,38.2,50.9,63.7$, and $76.4 \mathrm{kA} / \mathrm{m}$. In Fig. 5, the maximum longitudinal piezomagnetic coefficient (the modulus) is plotted as a function of the amplitude of $H_{\mathrm{ac}}$ for both samples CFOI and CFOA (the semilog 2 plot). It appears that dynamic magnetostriction, i.e., the piezomagnetic coefficient, depends on the amplitude of the excitation field. For both samples, the piezomagnetic coefficient $q^{\text {ac }}$ is enhanced by increasing the excitation field $H_{\mathrm{ac}}$ until it reaches an optimum field and then starts decreasing. Indeed, by increasing the field from 0.8 to $25.5 \mathrm{kA} / \mathrm{m}$, the ac piezomagnetic coefficient for CFOI is increased from 0.36 to $0.86 \mathrm{~nm} / \mathrm{A}$. For CFOA, the coefficient is increased from $0.30 \mathrm{~nm} / \mathrm{A}$ at $0.8 \mathrm{kA} / \mathrm{m}$ to $0.90 \mathrm{~nm} / \mathrm{A}$ at $50.9 \mathrm{kA} / \mathrm{m}$. Hence, a higher coefficient can be found for CFOA than for CFOI, as in quasistatic values, but requiring a higher ac field. The decrease of the piezomagnetic coefficient once a given $H_{\text {ac }}$ is reached is possibly due to the nonlinearity of the magnetostrictive curve. When the driving field is too strong, secondary harmonics are enhanced and hence reduce the fundamental signal (see Sec. III C). The dependence of the piezomagnetic coefficient to the excitation field can be explained by Eq. (2):

$$
\begin{aligned}
q^{\mathrm{ac}} & =\left(\frac{\partial \lambda_{\mathrm{ac}}}{\partial H_{\mathrm{ac}}}\right)_{H_{\mathrm{dc}}} \\
& =\left(\frac{\partial \lambda_{\mathrm{ac}}}{\partial M} \frac{\partial M}{\partial H_{\mathrm{ac}}}\right)_{H_{\mathrm{dc}}}, \\
q^{\mathrm{ac}} & =\left(\frac{\partial \lambda_{\mathrm{ac}}}{\partial M} \chi_{\mathrm{ac}}\right)_{H_{\mathrm{dc}}},
\end{aligned}
$$

where $\chi_{\mathrm{ac}}$ is defined as the response of the magnetization of the material to a small change in the magnetic field [61]. Thus, $q^{\text {ac }}$ depends on $H_{\mathrm{ac}}$ through the dependence of the dynamic susceptibility $\chi_{\mathrm{ac}}$ on $H_{\mathrm{ac}}$.

In Fig. 5, the piezomagnetic coefficients $q^{\text {ac }}$ are also compared to the strain derivative $q^{\mathrm{dc}}$. For CFOI, at $0.8 \mathrm{kA} / \mathrm{m}$, the dynamic coefficient is $30 \%$ of the quasistatic value and reaches $70 \%$ at $25.5 \mathrm{kA} / \mathrm{m}$. For CFOA, at $0.8 \mathrm{kA} / \mathrm{m}$, the dynamic coefficient is $20 \%$ of the quasistatic value and reaches $60 \%$ at $50.9 \mathrm{kA} / \mathrm{m}$. Moreover, $q^{\text {ac }}$ is enhanced by only $50 \%$ when the ac field is increased from 0.8 to $12.7 \mathrm{kA} / \mathrm{m}$ for CFOA, whereas $q^{\text {ac }}$ is increased by $100 \%$ for the same range of field for CFOI. This result confirms that materials with high permeability exhibit higher dynamic deformation at low excitation field $\left(H_{\mathrm{ac}} \leq 25.5 \mathrm{kA} / \mathrm{m}\right)$, even though they have a lower saturation magnetostriction $\lambda_{S}$ and a lower static derivative strain $\partial \lambda / \partial H_{\mathrm{dc}}$. On the other hand, at high driving field $\left(H_{\mathrm{ac}}>25.5 \mathrm{kA} / \mathrm{m}\right)$, CFOA enhances its coefficient by $100 \%$ and is kept high between 38.2 and $76.4 \mathrm{kA} / \mathrm{m}$, whereas CFOI increases by only $16 \%$ until decreasing drastically for $H_{\mathrm{ac}}>50.9 \mathrm{kA} / \mathrm{m}$. These results show that high anisotropy samples, i.e., those with low permeability, need a high driving field to be efficient in dynamic applications, but they have a better effect when excited at the optimum alternating field.

The same measurements are performed in the transverse direction $q_{21}^{\text {ac }}$ for both samples. However, because of the low dynamic deformation in this direction, weak signal voltage is measured, hence affecting the accuracy and making the measurements unreliable. As a first estimation, the transverse dynamic deformation is considered to keep the same ratio between the longitudinal and transverse coefficients as in the quasistatic deformation 
$\left[\left(q_{11}^{\mathrm{ac}}\right) /\left(q_{21}^{\mathrm{ac}}\right)\right]=\left[\left(q_{11}^{\mathrm{dc}}\right) /\left(q_{21}^{\mathrm{dc}}\right)\right]$, for any given bias field $H_{\mathrm{dc}}$. Hence, $q_{21}^{\text {ac }}$ is calculated using

$$
q_{21}^{\mathrm{ac}}=\left(\frac{q_{21}^{\mathrm{dc}}}{q_{11}^{\mathrm{dc}}} \cdot q_{11}^{\mathrm{ac}}\right)_{H_{\mathrm{dc}}}
$$

\section{Magnetoelectric effect}

Let us now focus on the magnetoelectric effect of the bilayers CFOI/PZT and CFOA/PZT. For several years now, authors have developed very accurate magnetoelectric models to describe this effect with respect to the material properties. Here, we use the low-frequency model for the transverse effect presented in Ref. [79]:

$\alpha_{31}=\frac{\eta\left(q_{11}^{m}+q_{21}^{m}\right) d_{31}^{e}}{\epsilon_{33}\left[\left(s_{11}^{e}+s_{21}^{e}\right)+\eta \gamma\left(s_{11}^{m}+s_{21}^{m}\right)\right]-2\left(d_{31}^{e}\right)^{2}}$,

where $\eta$ is the mechanical coupling factor, $d_{31}^{e}$ is the transverse piezoelectric coefficient, $\epsilon_{33}$ is the dielectric permittivity, $s_{i j}$ represents the compliances, and $\gamma=$ $\left[\left(\nu_{e}\right) /\left(\nu_{m}\right)\right]=\left[\left(t_{e}\right) /\left(t_{m}\right)\right]$ is the volume ratio, with $t_{e}$ and $t_{m}$ being the thicknesses of the PZT and CFO, respectively.

For the PZT (PZ27 from Ferroperm), we use the following parameters for calculations: $d_{31}^{e}=-170 \mathrm{pC} / \mathrm{N}$, $s_{11}^{e}=17 \mathrm{pm}^{2} / \mathrm{N}, s_{21}^{e}=-6.6 \mathrm{pm}^{2} / \mathrm{N}, \epsilon_{r 33}=1800$, and, for CFOA (the same as for CFOI), we use $s_{11}^{m}=6.44 \mathrm{pm}^{2} / \mathrm{N}$, $s_{21}^{m}=-1.96 \mathrm{pm}^{2} / \mathrm{N}$ [42]. The mechanical coupling factor $\eta$ can be defined as being the ratio of the average strain between the piezoelectric and magnetic phases $\left\langle S^{e}\right\rangle /\left\langle S^{m}\right\rangle$. FEM simulation is used to simulate the mechanical coupling factor. The simulation takes into account an epoxy layer of $30 \mu \mathrm{m}$ between the piezoelectric and magnetostrictive phases, and a friction coefficient $k=0.25$ is introduced at each interface, as explained in Ref. [80]. The resulting bilayer mechanical coupling factor is $\eta=0.25$.

In Fig. 6, we show the transverse magnetoelectric coefficient $\alpha_{31}^{\exp }(0.8 \mathrm{kA} / \mathrm{m})$ for the bilayer CFOA/PZT, measured at low frequency $(80 \mathrm{~Hz})$ and with a low-ac field $(0.8 \mathrm{kA} / \mathrm{m})$ as a function of the static field. Two theoretical magnetoelectric coefficients curves are also plotted in this figure. The first (the dotted line) is calculated using strain derivative coefficients $\alpha_{31}^{\text {th }}\left(q^{\mathrm{dc}}\right)$, whereas the second plot (the blue squares) is calculated using a piezomagnetic coefficient measured at $0.8 \mathrm{kA} / \mathrm{m}$ $\alpha_{31}^{\text {th }}\left[q^{\text {ac }}(0.8 \mathrm{kA} / \mathrm{m})\right]$. The second method provides a much more accurate curve than the first one. Indeed, a maximum magnetoelectric coefficient of $494 \mathrm{mV} / \mathrm{A}$ is calculated using the strain derivative $q^{\mathrm{dc}}$, whereas the experimental value is $100 \mathrm{mV} / \mathrm{A}$. By contrast, using the measured piezomagnetic coefficient $q^{\text {ac }}$ gives a maximum $\mathrm{ME}$ coefficient of $90 \mathrm{mV} / \mathrm{A}$. Hence, the relative error for $\alpha_{31}^{\text {th }}\left(q^{\mathrm{dc}}\right)$ is $394 \%$ with respect to the experimental value,

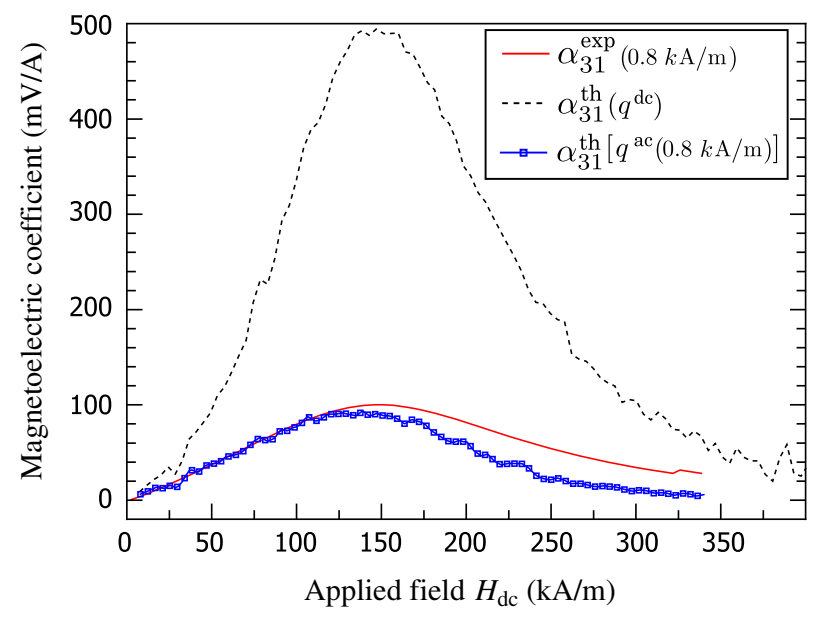

FIG. 6. Magnetoelectric coefficient measured for CFOA/PZT at $H_{\mathrm{ac}} \sim 0.8 \mathrm{kA} / \mathrm{m}$ and $80 \mathrm{~Hz}$, compared to the calculated coefficient integrating either the measured strain derivative $q^{\mathrm{dc}}$ or the piezomagnetic coefficient $q^{\text {ac }}$ at $0.8 \mathrm{kA} / \mathrm{m}$.

whereas it decreases to $10 \%$ with $\alpha_{31}^{\text {th }}\left[q^{\text {ac }}(0.8 \mathrm{kA} / \mathrm{m})\right]$. Even though good accuracy is found using dynamic deformation, the relative error (approximately 10\%) should be mainly attributed to $q_{21}^{\mathrm{ac}}$, which is calculated using a static ratio at any given bias field, as described in Sec. III B.

However, to improve the accuracy on the maximum magnetoelectric coefficient $\alpha_{31}^{\max }$, we use a more reliable method to estimate $q_{21}^{\text {ac }}$ from the measured values. In this case, the ratio between the transverse and longitudinal coefficients is chosen at the maximum strain derivative sum $q_{11}^{\mathrm{dc}}+q_{21}^{\mathrm{dc}}$. The longitudinal piezomagnetic coefficient is hence taken at its maximum value, giving

$$
q_{21}^{\mathrm{ac}}\left(\alpha_{31}^{\max }\right)=\left(\frac{q_{21}^{\mathrm{dc}}}{q_{11}^{\mathrm{dc}}}\right)_{\max \left(\mathrm{q}_{11}^{\mathrm{dc}}+\mathrm{q}_{21}^{\mathrm{dc}}\right)} q_{11 \max }^{\mathrm{ac}} .
$$

Hence, it is found that $q_{21}^{\mathrm{ac}}\left(\alpha_{31}^{\max }\right)=-0.125 q_{11 \max }^{\mathrm{ac}}$ for CFOA. In this case, a maximum $\alpha_{31}^{\text {th }}\left[q^{\text {ac }}(0.8 \mathrm{kA} / \mathrm{m})\right]$ value of $96 \mathrm{mV} / \mathrm{A}$ is found, thus reducing the relative error to $4 \%$. In the following, only the maximum magnetoelectric coefficient is considered, and the transverse dynamic coefficient $q_{21}^{\text {ac }}$ is therefore calculated using

$$
q_{21}^{\mathrm{ac}}\left(\alpha_{31}^{\max }\right)=-0.35 q_{11 \max }^{\mathrm{ac}}
$$

for CFOI, and

$$
q_{21}^{\mathrm{ac}}\left(\alpha_{31}^{\max }\right)=-0.125 q_{11 \max }^{\mathrm{ac}}
$$

for CFOA.

As the dynamic magnetostriction is a function of the amplitude of the ac field, a similar behavior is expected for the magnetoelectric effect. Hence, ME measurements on both bilayer CFOI/PZT and bilayer CFOA/PZT are performed for various $H_{\mathrm{ac}}$ values in the interval between 0.8 


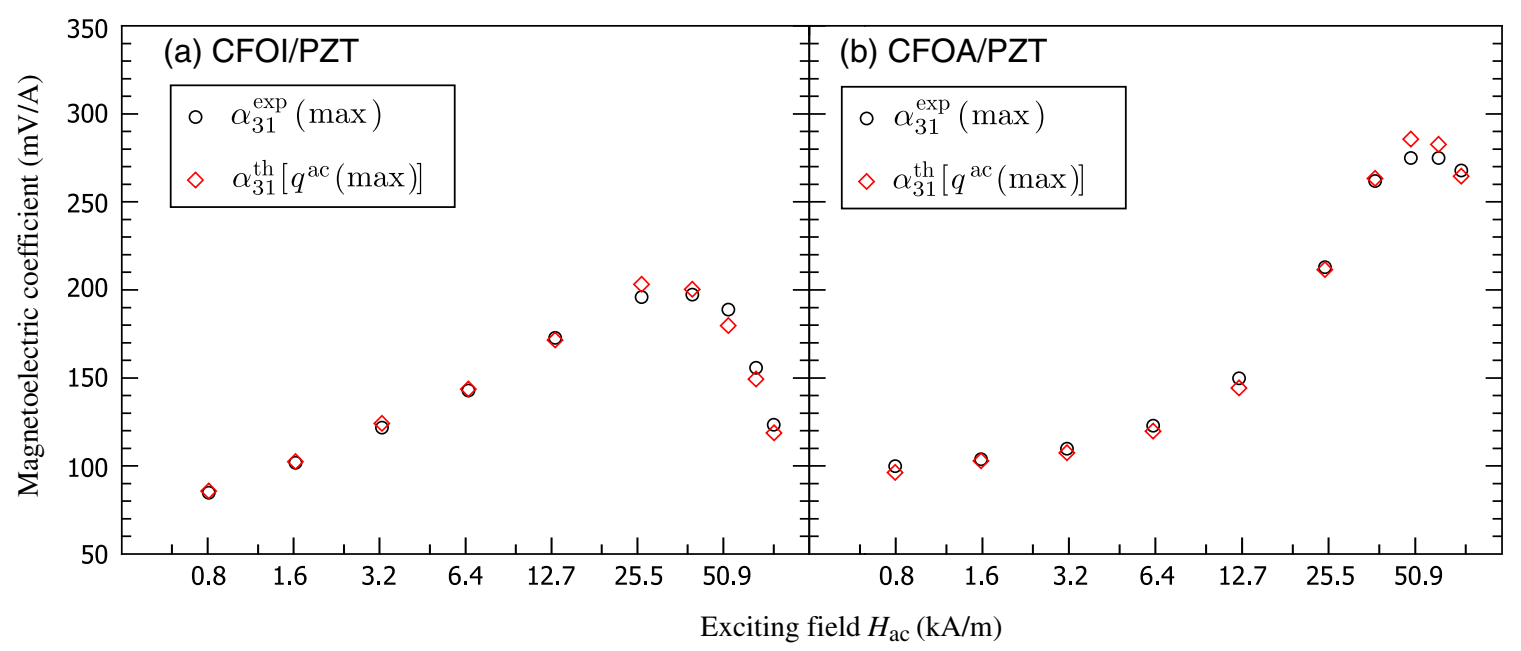

FIG. 7. The maximum magnetoelectric coefficient measured $\alpha_{31}^{\text {exp }}$ or calculated $\alpha_{31}^{\text {th }}$ for ME bilayers (a) CFOI/PZT and (b) CFOA/PZT as a function of the excitation field $H_{\mathrm{ac}}$ at low frequency $(80 \mathrm{~Hz})$ (the semilog $\mathrm{g}_{2}$ plot). The calculated values integrate the measured piezomagnetic coefficients $q^{\text {ac }}$ at each exciting field.

to $76.4 \mathrm{kA} / \mathrm{m}$. From these measurements, the maximum magnetoelectric coefficient reached is retrieved and plotted (the black open circles) as a function of the excitation field in Fig. 7. As expected, the magnetoelectric effect depends on the amplitude of $H_{\mathrm{ac}}$, showing a nonlinear magnetoelectric effect with respect to the ac field.

The ME effect tends to behave as with dynamic magnetostriction: an enhancement of the effect until reaching an optimum $H_{\mathrm{ac}}$ field and then a decrease of the signal. As with the piezomagnetic effect, the decrease following the maximum can be explained by the nonlinearity of the magnetostrictive curve due to the magnetic hysteretic conduct of the ceramic, leading to an increase of harmonics with an increasing $H_{\mathrm{ac}}$ [57,81-85]. To visualize this effect, we report in Fig. 8 the total harmonic distortion $\left(\mathrm{THD}_{F}\right)$ [86] calculated from the magnetoelectric voltage measured as a function of the ac field for both bilayers. It appears that

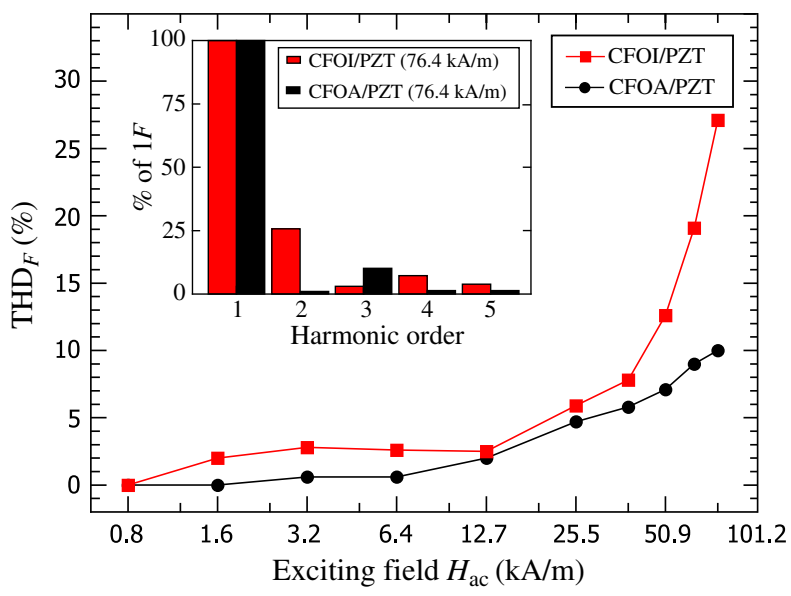

FIG. 8. Total harmonic distortion as a function of the excitation field for CFOI/PZT and CFOA/PZT (the semilog 2 plot). (Inset) The percentage of harmonics with respect to the fundamental for a driving field of $76.4 \mathrm{kA} / \mathrm{m}$. when the excitation field $H_{\mathrm{ac}}$ is increased, the $\mathrm{THD}_{F}$ increases. For CFOI/PZT, the ME effect stops increasing at $38.2 \mathrm{kA} / \mathrm{m}$ where the $\mathrm{THD}_{F}$ is $7.8 \%$, and for CFOA/ PZT stops increasing at $50.9 \mathrm{kA} / \mathrm{m}$ where the $\mathrm{THD}_{F}$ is 7.1\%. However, the ME coefficient for CFOI/PZT decreases drastically at 63.7 and $76.4 \mathrm{kA} / \mathrm{m}$, where the $\mathrm{THD}_{F}$ is equal to $19.1 \%$ and $27.1 \%$, respectively. On the other hand, the $\mathrm{THD}_{F}$ is still quite low for CFOA/PZT at $76.4 \mathrm{kA} / \mathrm{m}$ (about 10\%). This low value might be due to the higher permeability of CFOI compared to CFOA. Materials with higher permeability require a lower field to reach the maximum dynamic deformation. Hence, by applying a strong ac field, the field is high enough to achieve the nonlinear part of the magnetostrictive curve, which mainly contributes to the increase of the second harmonic [85]. This explanation is highlighted in the inset Fig. 8, which shows the percentage of harmonic distortion with respect to the fundamental when excited at $76.4 \mathrm{kA} / \mathrm{m}$. The contribution of the second harmonic in CFOI/PZT is high (approximately 25\%), whereas the major contribution of distortion in CFOA/PZT is from the third harmonic (about 10\%).

Figure 7 also shows that, for the 1.6 to $12.7 \mathrm{kA} / \mathrm{m}$ ac fields, CFOI/PZT has a better magnetoelectric effect than CFOA/PZT. The bilayer composed of ferrite with higher permeability is more efficient at low excitation field than the ferrite with lower permeability. However, for a $25.5 \mathrm{kA} / \mathrm{m}$ or higher $H_{\mathrm{ac}}, \mathrm{CFOA} / \mathrm{PZT}$ reaches much higher values than $\mathrm{CFOI} / \mathrm{PZT}$. Indeed, the maximum ME coefficient achieved for isotropic CFO is $198 \mathrm{mV} / \mathrm{A}$, while it reaches $275 \mathrm{mV} / \mathrm{A}$ for the anisotropic sample. In diamond symbols, the calculated maximum magnetoelectric coefficients using the previously measured piezomagnetic coefficients are plotted (the red open diamonds). For both samples, good accuracy between the experimental and calculated data is found, with a low relative error 


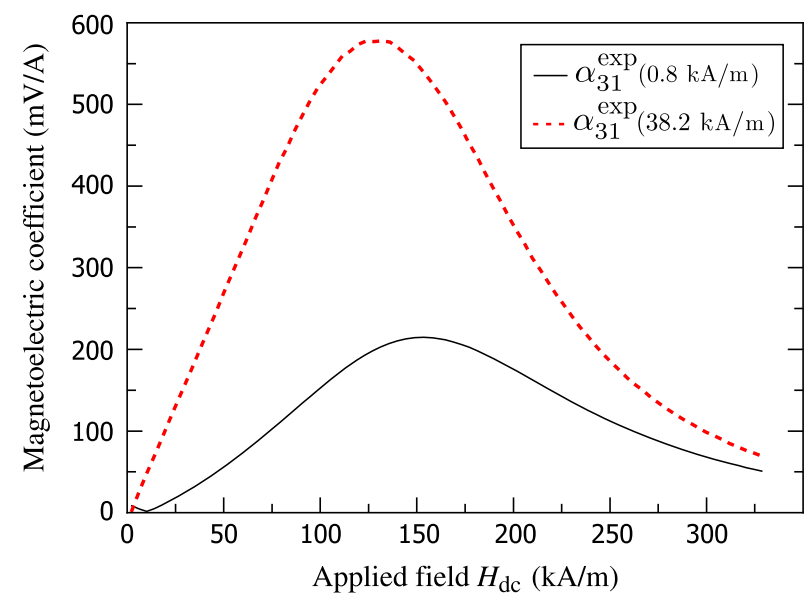

FIG. 9. Magnetoelectric coefficient measured for the trilayer CFOA/PZT/CFOA at the exciting field $H_{\mathrm{ac}}$ of 0.8 and $38.2 \mathrm{kA} / \mathrm{m}$ and at low frequency $(80 \mathrm{~Hz})$.

$(<5 \%)$. This accuracy proves the relevance of using the piezomagnetic coefficient instead of quasistatic strain derivative coefficients to model correctly the ME effect, and consequently to any dynamic magnetostrictive applications with cobalt ferrite. It also demonstrates that using semihard anisotropic cobalt ferrite with high static deformation is not necessarily more efficient than using the soft isotropic one, mainly because dynamic deformation, i.e., the ME effect, needs high permeability to have good performance for low amplitudes of $H_{\mathrm{ac}}$. However, it seems that semihard CFO ferrite exhibits much higher linearity in response to the amplitude of $H_{\mathrm{ac}}$, which could be interesting if used in a magnetic or current environment exhibiting high-amplitude signals.

In order to evaluate the maximum potential of the composite CFOA/PZT at optimum alternating field, a ME trilayer with an optimized $\mathrm{PZT/CFO}$ volume ratio is made. Trilayer composite is known to enhance the mechanical coupling factor and reduce the demagnetizing effect, which contributes to enhancing the magnetoelectric effect [80]. In Fig. 9, the magnetoelectric effect of CFOA/ PZT/CFOA is shown for a low excitation field $0.8 \mathrm{kA} / \mathrm{m}$ and the optimum ac field $38.2 \mathrm{kA} / \mathrm{m}$, at low frequency $(80 \mathrm{~Hz})$. A maximum $\alpha_{31}$ of $578 \mathrm{mV} / \mathrm{A}$ (about $460 \mathrm{mV} / \mathrm{cm} \mathrm{Oe}$ ) at $38.2 \mathrm{kA} / \mathrm{m}$ is reached, which is 3 times higher than the value measured at $0.8 \mathrm{kA} / \mathrm{m}$ (approximately $193 \mathrm{mV} / \mathrm{A}$ ). This result points out that it is worth characterizing semihard materials at high excitation field to be able to use their maximum potential in dynamic applications. This coefficient could be increased by using a more efficient piezoelectric materials as lead magnesium niobate-lead titanate (PMN-PT) or lead zirconium niobate-lead titanate (PZN-PT) single crystals [87]. It could also be enhanced by cosintering both magnetic and piezoelectric phases instead of bonding them, hence increasing the mechanical coupling. The cosintering was recently achieved in bulk $\mathrm{CFO} / \mathrm{BaTiO}_{3}$ composites using SPS [49]. One can also notice the very linear aspect of the curve between 0 and $75 \mathrm{kA} / \mathrm{m}$, which is a predominant parameter to produce a precise dc current sensor using the ME effect $[85,88]$. This linearity could also be exploited for energy-harvesting purpose with an extremely low-frequency and high-ac field [89].

\section{CONCLUSION}

In summary, we investigate in this paper the dynamic magnetostriction of isotropic and anisotropic cobalt ferrite. The measured piezomagnetic coefficient is found to be much lower than the quasistatic strain derivative coefficient. Also, it is shown that piezomagnetic coefficients depend on the amplitude of the driving ac field and behave nonlinearly with it. As the isotropic sample has higher permeability, it exhibits higher dynamic deformation than the anisotropic sample for a low-ac field, whereas the strain derivative coefficient is higher for the anisotropic sample. However, at very high driving field $(>38.2 \mathrm{kA} / \mathrm{m})$, the anisotropic CFO shows higher dynamic deformation. This work shows that the strain derivative is not the appropriate parameter to describe dynamic deformation for semihard materials such as cobalt ferrite. Our results will be useful to properly characterize other low-permeability materials such as $\mathrm{TbFe}_{2}, \mathrm{DyFe}_{2}, \mathrm{SmFe}_{2}$, and Terfenol- $D$.

The magnetoelectric effect is also investigated for these two ceramics, showing a general behavior which can be correlated with dynamic magnetostriction. The effect is dependent on the ac field, and good accuracy is found between predicted and experimental data when piezomagnetic coefficients are introduced in the ME model instead of quasistatic strain derivatives. Hence, using an optimizedgeometry ME trilayer allows us to reach a high ME coefficient of $578 \mathrm{mV} / \mathrm{A}$ for a high-ac field of $38.2 \mathrm{kA} / \mathrm{m}$ at low frequency $(80 \mathrm{~Hz})$. This result points out the limitations of cobalt ferrite in magnetoelectric applications for a low-ac field but shows its great potential for applications in a highac field.

[1] É. Du Trémolet de Lacheisserie, Magnetostriction, Theory and Applications of Magnetoelasticity (CRC Press, Boca Raton, 1993).

[2] D.C. Jiles, Theory of the magnetomechanical effect, J. Phys. D 28, 1537 (1995).

[3] Y. K. Fetisov, D. D. Chashin, N. A. Ekonomov, D. A. Burdin, and L. Y. Fetisov, Correlation between magnetoelectric and magnetic properties of ferromagneticpiezoelectric structures, IEEE Trans. Magn. 51, 1 (2015).

[4] M. Anjanappa and Yuefei Wu, Magnetostrictive particulate actuators: Configuration, modeling and characterization, Smart Mater. Struct. 6, 393 (1997).

[5] S. Karunanidhi and M. Singaperumal, Design, analysis and simulation of magnetostrictive actuator and its application to high dynamic servo valve, Sens. Actuators A 157, 185 (2010). 
[6] Youngkyu Kim and Yoon Young Kim, A novel Terfenol- $D$ transducer for guided-wave inspection of a rotating shaft, Sens. Actuators A 133, 447 (2007).

[7] F. Claeyssen, N. Lhermet, R. L. Le Letty, and P. Bouchilloux, Actuators, transducers and motors based on giant magnetostrictive materials, J. Alloys Compd. 258, 61 (1997).

[8] A. G. Olabi and A. Grunwald, Design and application of magnetostrictive materials, Mater. Des. 29, 469 (2008).

[9] A. Al-Hajjeh, E. Lynch, C. T. Law, and R. El-Hajjar, Characteristics of a magnetostrictive composite stress sensor, IEEE Magn. Lett. 7, 1 (2016).

[10] R. Sato Turtelli, M. Kriegisch, M. Atif, and R. Grossinger, Co-ferrite-A material with interesting magnetic properties, IOP Conf. Ser. Mater. Sci. Eng. 60, 012020 (2014).

[11] C. C. H. Lo, A. P. Ring, J. E. Snyder, and D. C. Jiles, Improvement of magnetomechanical properties of cobalt ferrite by magnetic annealing, IEEE Trans. Magn. 41, 3676 (2005).

[12] Atif Muhammad, Reiko Sato-Turtelli, Martin Kriegisch, Roland Grossinger, Rssinger, Frank Kubel, and Thomas Konegger, Large enhancement of magnetostriction due to compaction hydrostatic pressure and magnetic annealing in $\mathrm{CoFe}_{2} \mathrm{O}_{4}$, J. Appl. Phys. 111, 013918 (2012).

[13] K. Khaja Mohaideen and P. A. Joy, High magnetostriction and coupling coefficient for sintered cobalt ferrite derived from superparamagnetic nanoparticles, Appl. Phys. Lett. 101, 072405 (2012).

[14] Y. X. Zheng, Q. Q. Cao, C. L. Zhang, H. C. Xuan, L. Y. Wang, D. H. Wang, and Y.W. Du, Study of uniaxial magnetism and enhanced magnetostriction in magneticannealed polycrystalline $\mathrm{CoFe}_{2} \mathrm{O}_{4}$, J. Appl. Phys. 110, 043908 (2011).

[15] Yuqiang Dai, Qianqian Gao, Jianming Dai, Xianwu Tang, Liguo Yang, Chaojun Cui, Xianchang Li, Chengbo Li, and Zhendong Liu, Influence of magnetic annealing on the ferroelectric and dielectric properties of $\mathrm{BaTiO}_{3}-\mathrm{CoFe}_{2} \mathrm{O}_{4}$ multilayer films prepared by chemical solution deposition, J. Alloys Compd. 672, 536 (2016).

[16] Jiquan Wang, Xuexu Gao, Chao Yuan, Jiheng Li, and Xiaoqian Bao, Magnetostriction properties of oriented polycrystalline $\mathrm{CoFe}_{2} \mathrm{O}_{4}$, J. Magn. Magn. Mater. 401, 662 (2016).

[17] Monaji Vinitha Reddy, Abdellah Lisfi, Sabin Pokharel, and Dibakar Das, Colossal piezomagnetic response in magnetically pressed $\mathrm{Zr}^{+4}$ substituted cobalt ferrites, Sci. Rep. 7, 7935 (2017).

[18] A. Aubert, V. Loyau, F. Mazaleyrat, and M. LoBue, Uniaxial anisotropy and enhanced magnetostriction of $\mathrm{CoFe}_{2} \mathrm{O}_{4}$ induced by reaction under uniaxial pressure with SPS, J. Eur. Ceram. Soc. 37, 3101 (2017).

[19] P. N. Anantharamaiah and P. A. Joy, Enhancing the strain sensitivity of $\mathrm{CoFe}_{2} \mathrm{O}_{4}$ at low magnetic fields without affecting the magnetostriction coefficient by substitution of small amounts of $\mathrm{Mg}$ for Fe, Phys. Chem. Chem. Phys. 18, 10516 (2016).

[20] P. N. Anantharamaiah and P. A. Joy, Tuning of the magnetostrictive properties of cobalt ferrite by forced distribution of substituted divalent metal ions at different crystallographic sites, J. Appl. Phys. 121, 093904 (2017).
[21] P. N. Anantharamaiah and P. A. Joy, Magnetic and magnetostrictive properties of aluminium substituted cobalt ferrite synthesized by citrate-gel method, J. Mater. Sci. 50, 6510 (2015).

[22] I. C. Nlebedim and D. C. Jiles, Suitability of cation substituted cobalt ferrite materials for magnetoelastic sensor applications, Smart Mater. Struct. 24, 025006 (2015).

[23] G. S. N. Rao, O. F. Caltun, K. H. Rao, P. S. V. Subba Rao, and B. Parvatheeswara Rao, Improved magnetostrictive properties of Co-Mn ferrites for automobile torque sensor applications, J. Magn. Magn. Mater. 341, 60 (2013).

[24] Chien-Yie Tsay, Yi-Hsiang Lin, and Shien-Uang Jen, Magnetic, magnetostrictive, and AC impedance properties of manganese substituted cobalt ferrites, Ceram. Int. 41, 5531 (2015).

[25] Xi Guoxi and Xi Yuebin, Effects on magnetic properties of different metal ions substitution cobalt ferrites synthesis by sol-gel auto-combustion route using used batteries, Mater. Lett. 164, 444 (2016).

[26] B. Chandra Sekhar, G. S. N. Rao, O. F. Caltun, B. Dhana Lakshmi, B. Parvatheeswara Rao, and P. S. V. Subba Rao, Magnetic and magnetostrictive properties of $\mathrm{Cu}$ substituted Co-ferrites, J. Magn. Magn. Mater. 398, 59 (2016).

[27] Nalla Somaiah, Tanjore V. Jayaraman, P. A. Joy, and Dibakar Das, Magnetic and magnetoelastic properties of Zn-doped cobalt-ferrites- $\mathrm{CoFe}_{2-x} \mathrm{Zn}_{x} \mathrm{O}_{4}(x=0,0.1,0.2$, and 0.3), J. Magn. Magn. Mater. 324, 2286 (2012).

[28] P. N. Anantharamaiah and P. A. Joy, High magnetostriction parameters of sintered and magnetic field annealed Ga-substituted $\mathrm{CoFe}_{2} \mathrm{O}_{4}$, Mater. Lett. 192, 169 (2017).

[29] Vinitha Monaji Reddy and Dibakar Das, Influence of $\mathrm{Zr}$ doping on the structural, magnetic and magnetoelastic properties of cobalt-ferrites, J. Alloys Compd. 634, 99 (2015).

[30] R. Ramchandra Kiran, R. A. Mondal, Sandhya Dwevedi, and G. Markandeyulu, Structural, magnetic and magnetoelectric properties of $\mathrm{Nb}$ substituted cobalt ferrite, J. Alloys Compd. 610, 517 (2014).

[31] P. N. Anantharamaiah and P. A. Joy, Effect of size and site preference of trivalent non-magnetic metal ions $\left(\mathrm{Al}^{3+}, \mathrm{Ga}^{3+}\right.$, $\mathrm{In}^{3+}$ ) substituted for $\mathrm{Fe}^{3+}$ on the magnetostrictive properties of sintered $\mathrm{CoFe}_{2} \mathrm{O}_{4}$, J. Phys. D 50, 435005 (2017).

[32] G. Bulai, L. Diamandescu, I. Dumitru, S. Gurlui, M. Feder, and O.F. Caltun, Effect of rare earth substitution in cobalt ferrite bulk materials, J. Magn. Magn. Mater. 390, 123 (2015).

[33] Shekhar D. Bhame and P. A. Joy, Magnetoelastic properties of terbium substituted cobalt ferrite, Chem. Phys. Lett. 685, 465 (2017).

[34] Li Yang, Guoxi Xi, Tianjun Lou, Xinsheng Wang, Jingjing Wang, and Yuanheng He, Preparation and magnetic performance of $\mathrm{Co}_{0.8} \mathrm{Fe}_{2.2} \mathrm{O}_{4}$ by a sol-gel method using cathode materials of spent Li-ion batteries, Ceram. Int. 42, 1897 (2016).

[35] Lu Yao, Yuebin Xi, Guoxi Xi, and Yong Feng, Synthesis of cobalt ferrite with enhanced magnetostriction properties by the sol-gel-hydrothermal route using spent Li-ion battery, J. Alloys Compd. 680, 73 (2016).

[36] Guoxi Xi, Lu Wang, and Tingting Zhao, Magnetic and magnetostrictive properties of RE-doped $\mathrm{Cu}-\mathrm{Co}$ ferrite fabricated from spent lithium-ion batteries, J. Magn. Magn. Mater. 424, 130 (2017). 
[37] Jungho Ryu, Shashank Priya, Kenji Uchino, and Hyoun-Ee Kim, Magnetoelectric effect in composites of magnetostrictive and piezoelectric materials, J. Electroceram. 8, 107 (2002).

[38] M. Fiebig, Revival of the magnetoelectric effect, J. Phys. D 38, R123 (2005).

[39] W. Eerenstein, N. D. Mathur, and J. F. Scott, Multiferroic and magnetoelectric materials, Nature (London) 442, 759 (2006).

[40] Z. Chu, H. Shi, M. J. Pourhosseiniasl, J. Wu, W. Shi, X. Gao, X. Yuan, and S. Dong, A magnetoelectric flux gate: New approach for weak DC magnetic field detection, Sci. Rep. 7, 8592 (2017).

[41] Jianxin Shen, Junzhuang Cong, Yisheng Chai, Dashan Shang, Shipeng Shen, Kun Zhai, Ying Tian, and Young Sun, Nonvolatile Memory Based on Nonlinear Magnetoelectric Effects, Phys. Rev. Applied 6, 021001 (2016).

[42] A. Aubert, V. Loyau, F. Mazaleyrat, and M. LoBue, Enhancement of the magnetoelectric effect in multiferroic $\mathrm{CoFe}_{2} \mathrm{O}_{4} / \mathrm{PZT}$ bilayer by induced uniaxial magnetic anisotropy, IEEE Trans. Magn. 53, 1 (2017).

[43] A. G. Boni, C. Chirila, I. Pasuk, R. Negrea, I. Pintilie, and L. Pintilie, Steplike Switching in Symmetric $\mathrm{PbZr}_{0.2} \mathrm{Ti}_{0.8} \mathrm{O}_{3} /$ $\mathrm{CoFe}_{2} \mathrm{O}_{4} / \mathrm{PbZr}_{0.2} \mathrm{Ti}_{0.8} \mathrm{O}_{3}$ Heterostructures for Multistate Ferroelectric Memory, Phys. Rev. Applied 8, 034035 (2017).

[44] Haibo Yang, Jintao Zhang, Ying Lin, and Tong Wang, High Curie temperature and enhanced magnetoelectric properties of the laminated $\mathrm{Li}_{0.058}\left(\mathrm{Na}_{0.535} \mathrm{~K}_{0.48}\right)_{0.942} \mathrm{NbO}_{3} /$ $\mathrm{Co}_{0.6} \mathrm{Zn}_{0.4} \mathrm{Fe}_{1.7} \mathrm{Mn}_{0.3} \mathrm{O}_{4}$ composites, Sci. Rep. 7, 44855 (2017).

[45] Yaru Wang, Yongping $\mathrm{Pu}, \mathrm{Yu}$ Shi, and Yongfei Cui, Ferroelectric, magnetic, magnetoelectric properties of the $\mathrm{Ba}_{0.9} \mathrm{Ca}_{0.1} \mathrm{Ti}_{0.9} \mathrm{Zr}_{0.1} \mathrm{O}_{3} / \mathrm{CoFe}_{2} \mathrm{O}_{4}$ laminated composites, J. Mater. Sci. 28, 11125 (2017).

[46] Haibo Yang, Ge Zhang, and Ying Lin, Enhanced magnetoelectric properties of the laminated $\mathrm{BaTiO}_{3}-\mathrm{CoFe}_{2} \mathrm{O}_{4}$ composites, J. Alloys Compd. 644, 390 (2015).

[47] M. Khodaei, Ahmad Eshghinejad, S. A. Seyyed Ebrahimi, and Sunggi Baik, Nanoscale magnetoelectric coupling study in (111)-oriented PZT-Co ferrite multiferroic nanobilayer thin film using piezoresponse force microscopy: Effect of Co ferrite composition, Sens. Actuators A 242, 92 (2016).

[48] Jiyong Fu, Washington Santa Rosa, Jean Claude M'Peko, Miguel Alguer, and Michel Venet, Magnetoelectric coupling in lead-free piezoelectric $\mathrm{Li}_{x}\left(\mathrm{~K}_{0.5} \mathrm{Na}_{0.5}\right)_{1-x} \mathrm{Nb}_{1-y} \mathrm{Ta}_{y} \mathrm{O}_{3}$ and magnetostrictive $\mathrm{CoFe}_{2} \mathrm{O}_{4}$ laminated composites, Phys. Lett. A 380, 1788 (2016).

[49] Yang Liu, Guowang Xu, Yongyu Xie, Hui Lv, Chuyun Huang, Yiwan Chen, Zhengfu Tong, Jing Shi, and Rui Xiong, Magnetoelectric behaviors in $\mathrm{BaTiO}_{3} / \mathrm{CoFe}_{2} \mathrm{O}_{4} /$ $\mathrm{BaTiO}_{3}$ laminated ceramic composites prepared by spark plasma sintering, Ceram. Int., DOI: 10.1016/j.ceramint .2018.02.192 (2018).

[50] Pei Peng, Ya-Ya Hu, Yong Liu, Shi Chen, Jing Shi, Rui Xiong, and Yue Zhang, Magnetoelectric effect of $\mathrm{CoFe}_{2} \mathrm{O}_{4} / \mathrm{Pb}(\mathrm{Zr}, \mathrm{Ti}) \mathrm{O}_{3}$ composite ceramics sintered via spark plasma sintering technology, Ceram. Int. 41, 6676 (2015).
[51] Ding Ai, Jianmei Xu, Can Huang, Wei Zhou, Ling Zhao, Jian Sun, and Qing Wang, Synthesis and magnetoelectric properties of multiferroic composites of lead lanthanum zirconate titanate and mesoporous cobalt ferrite, Scr. Mater. 136, 29 (2017).

[52] M. Rasly, M. Afifi, A. E. Shalan, and M. M. Rashad, A quantitative model based on an experimental study for the magnetoelectric coupling at the interface of cobalt ferrite-barium titanate nanocomposites, Appl. Phys. A 123, 331 (2017).

[53] Mukesh Kumari, Chandra Prakash, and Ratnamala Chatterjee, Room temperature large self-biased magnetoelectric effect in non-lead based piezoelectric and magnetostrictive (0-3) particulate composite system, J. Magn. Magn. Mater. 429, 60 (2017).

[54] Yang Liu, Guowang Xu, Hui Lv, Chuyun Huang, Yiwan Chen, Yeguang Bie, Jinye Zhang, Ting Liu, Ni Hu, Gang Deng, Ling Pei, Jing Shi, and Rui Xiong, Magnetoelectric ceramic composites prepared by spark plasma sintering with notably enhanced magnetoelectric effect, J. Mater. Sci. 28, 3746 (2017).

[55] M. Naveed-Ul-Haq, Vladimir V. Shvartsman, Gabriel Constantinescu, Harsh Trivedi, Soma Salamon, Joachim Landers, Heiko Wende, and Doru C. Lupascu, Effect of $\mathrm{Al}^{3+}$ modification on cobalt ferrite and its impact on the magnetoelectric effect in BCZT-CFO multiferroic composites, J. Mater. Sci. 52, 13402 (2017).

[56] Hao-Miao Zhou, Xiao-Wei Ou, Ying Xiao, Shao-Xing Qu, and Hua-Ping $\mathrm{Wu}, \mathrm{An}$ analytical nonlinear magnetoelectric coupling model of laminated composites under combined pre-stress and magnetic bias loadings, Smart Mater. Struct. 22, 035018 (2013).

[57] Yang Shi and Yuan-Wen Gao, Theoretical study on nonlinear magnetoelectric effect and harmonic distortion behavior in laminated composite, J. Alloys Compd. 646, 351 (2015).

[58] Shunzu Zhang, Hong Yao, and Yuanwen Gao, A 2D mechanical-magneto-thermal model for direction-dependent magnetoelectric effect in laminates, J. Magn. Magn. Mater. 428, 437 (2017).

[59] G. Srinivasan, E. T. Rasmussen, J. Gallegos, R. Srinivasan, Yu. I. Bokhan, and V. M. Laletin, Magnetoelectric bilayer and multilayer structures of magnetostrictive and piezoelectric oxides, Phys. Rev. B 64, 214408 (2001).

[60] S. Chikazumi, Physics of Ferromagnetism (Oxford University Press, Oxford, 2009), p. 11.

[61] M. Bałanda, H.-A. Krug von Nidda, M. Heinrich, and A. Loidl, in Relaxation Phenomena, edited by Wolfgang Haase and Stanislaw Wróbel (Springer, Berlin, 2003), p. 90.

[62] J. Fialka and P. Benes, in Proceedings of the 2012 IEEE International Instrumentation and Measurement Technology Conference, Graz, Austria, 2012 (IEEE, New York, 2012), p. 37.

[63] J. Hudson, S. C. Busbridge, and A. R. Piercy, Dynamic magneto-mechanical properties of epoxy-bonded Terfenol$D$ composites, Sens. Actuators A 81, 294 (2000).

[64] S. W. Or, N. Nersessian, G. P. McKnight, and G. P. Carman, Dynamic magnetomechanical properties of [112]-oriented Terfenol-D/epoxy 1-3 magnetostrictive particulate composites, J. Appl. Phys. 93, 8510 (2003). 
[65] Siu Wing Or, N. Nersessian, and G. P. Carman, Dynamic magnetomechanical behavior of Terfenol-D/epoxy 1-3 particulate composites, IEEE Trans. Magn. 40, 71 (2004).

[66] F. Claeyssen, D. Colombani, A. Tessereau, and B. Ducros, Giant dynamic magnetostrain in rare earth-iron magnetostrictive materials, IEEE Trans. Magn. 27, 5343 (1991).

[67] L. Bian, Y. Wen, and P. Li, Dynamic magnetomechanical behavior of $\mathrm{Tb}_{x} \mathrm{Dy}_{1-x} \mathrm{Fe}_{y}$ alloy under small-signal $\mathrm{AC}$ drive fields superposed with various bias fields, IEEE Trans. Magn. 52, 1 (2016).

[68] Caijiang Lu, Ping Li, Yumei Wen, Aichao Yang, Chao Yang, Jin Yang, Wei He, Jitao Zhang, and Wenli Li, Dynamic magnetostrictive properties of magnetizationgraded ferromagnetic material and application in magnetoelectric composite, J. Appl. Phys. 115, 17C726 (2014).

[69] B. Kundys, Yu. Bukhantsev, S. Vasiliev, D. Kundys, M. Berkowski, and V. P. Dyakonov, Three terminal capacitance technique for magnetostriction and thermal expansion measurements, Rev. Sci. Instrum. 75, 2192 (2004).

[70] G. Srinivasan, Magnetoelectric composites, Annu. Rev. Mater. Res. 40, 153 (2010).

[71] R. M. Bozorth and J. G. Walker, Magnetostriction of single crystals of cobalt and nickel ferrites, Phys. Rev. 88, 1209 (1952).

[72] G. Srinivasan, E. T. Rasmussen, and R. Hayes, Magnetoelectric effects in ferrite-lead zirconate titanate layered composites: The influence of zinc substitution in ferrites, Phys. Rev. B 67, 014418 (2003).

[73] V. Loyau, V. Morin, J. Fortineau, M. LoBue, and F. Mazaleyrat, A method to decrease the harmonic distortion in $\mathrm{Mn}-\mathrm{Zn}$ ferrite/PZT and Ni-Zn ferrite/PZT layered composite rings exhibiting high magnetoelectric effects, J. Appl. Phys. 118, 154101 (2015).

[74] U. Laletin, G. Sreenivasulu, V. M. Petrov, T. Garg, A. R. Kulkarni, N. Venkataramani, and G. Srinivasan, Hysteresis and remanence in magnetoelectric effects in functionally graded magnetostrictive-piezoelectric layered composites, Phys. Rev. B 85, 104404 (2012).

[75] Ru Zhang, Lei Jin, Gaojian Wu, and Ning Zhang, Magnetic force driven magnetoelectric effect in Mn-Zn-ferrite/PZT composites, Appl. Phys. Lett. 110, 112901 (2017).

[76] X. Zhuang, C. M. Leung, G. Sreenivasulu, M. Gao, J. Zhang, G. Srinivasan, J. Li, and D. Viehland, Upper limit for power conversion in magnetoelectric gyrators, Appl. Phys. Lett. 111, 163902 (2017).

[77] G. Srinivasan, R. Hayes, C. P. DeVreugd, V. M. Laletsin, and N. Paddubnaya, Dynamic magnetoelectric effects in bulk and layered composites of cobalt zinc ferrite and lead zirconate titanate, Appl. Phys. A 80, 891 (2005).

[78] J. E. Goldman, New techniques and results in the measurement of magnetostriction, J. Phys. Radium 12, 471 (1951).

[79] V. Loyau, V. Morin, G. Chaplier, M. LoBue, and F. Mazaleyrat, Magnetoelectric effect in layered ferrite/PZT composites. Study of the demagnetizing effect on the magnetoelectric behavior, J. Appl. Phys. 117, 184102 (2015).

[80] V. Loyau, A. Aubert, M. LoBue, and F. Mazaleyrat, Analytical modeling of demagnetizing effect in magnetoelectric ferrite/PZT/ferrite trilayers taking into account a mechanical coupling, J. Magn. Magn. Mater. 426, 530 (2017).

[81] K. E. Kamentsev, Y. K. Fetisov, and G. Srinivasan, Lowfrequency nonlinear magnetoelectric effects in a ferritepiezoelectric multilayer, Appl. Phys. Lett. 89, 142510 (2006).

[82] D. A. Burdin, D. V. Chashin, N. A. Ekonomov, L. Y. Fetisov, Y. K. Fetisov, G. Sreenivasulu, and G. Srinivasan, Nonlinear magneto-electric effects in ferromagnetic-piezoelectric composites, J. Magn. Magn. Mater. 358-359, 98 (2014).

[83] Hao Xu, Yongmao Pei, Daining Fang, and Panding Wang, Nonlinear harmonic distortion effect in magnetoelectric laminate composites, Appl. Phys. Lett. 105, 012904 (2014).

[84] D. A. Burdin, D. V. Chashin, N. A. Ekonomov, and Y. K. Fetisov, Static deformation of a ferromagnet in alternating magnetic field, J. Magn. Magn. Mater. 406, 217 (2016).

[85] D. Burdin, D. Chashin, N. Ekonomov, L. Fetisov, Y. Fetisov, and M. Shamonin, DC magnetic field sensing based on the nonlinear magnetoelectric effect in magnetic heterostructures, J. Phys. D 49, 375002 (2016).

[86] D. Shmilovitz, On the definition of total harmonic distortion and its effect on measurement interpretation, IEEE Transactions on Power Delivery 20, 526 (2005).

[87] Zhengbao Yang and Jean Zu, Comparison of PZN-PT, PMN-PT single crystals and PZT ceramic for vibration energy harvesting, Energy Conversion and Management 122, 321 (2016).

[88] M. Bichurin, R. Petrov, V. Leontiev, G. Semenov, and O. Sokolov, Magnetoelectric current sensors, Sensors 17, 1271 (2017).

[89] T. Lafont, L. Gimeno, J. Delamare, G. A. Lebedev, D. I. Zakharov, B. Viala, O. Cugat, N. Galopin, L. Garbuio, and O. Geoffroy, Magnetostrictive-piezoelectric composite structures for energy harvesting, J. Micromech. Microeng. 22, 094009 (2012). 